PROCEEDINGS OF THE

AMERICAN MATHEMATICAL SOCIETY

Volume 140, Number 3, March 2012, Pages 755-763

S 0002-9939(2011)11024-4

Article electronically published on July 6, 2011

\title{
INVARIANCE UNDER TWISTING FOR CROSSED PRODUCTS
}

\author{
FLORIN PANAITE
}

(Communicated by Gail R. Letzter)

\begin{abstract}
We prove a result of the type "invariance under twisting" for Brzeziński's crossed products as a common generalization of the invariance under twisting for twisted tensor products of algebras and the invariance under twisting for quasi-Hopf smash products. It turns out that this result contains also as a particular case the equivalence of crossed products by a coalgebra (due to Brzeziński).
\end{abstract}

\section{INTRODUCTION}

If $A$ and $B$ are (associative unital) algebras and $R: B \otimes A \rightarrow A \otimes B$ is a linear map satisfying certain axioms (such an $R$ is called a twisting map), then $A \otimes B$ becomes an associative unital algebra with a multiplication defined in terms of $R$ and the multiplications of $A$ and $B$; this algebra structure on $A \otimes B$ is denoted by $A \otimes_{R} B$ and is called the twisted tensor product of $A$ and $B$ afforded by $R$ (cf. [4, 7]). This construction appeared in a number of contexts and has various applications; see [6] for a detailed discussion and references. Moreover, there are many concrete examples of twisted tensor products, such as the Hopf smash product and other kinds of products arising in Hopf algebra theory.

A very general result about twisted tensor products of algebras was obtained in [6. It was directly inspired by the invariance under twisting of the Hopf smash product (and thus it was called invariance under twisting for twisted tensor products of algebras), but it contains also as particular cases a number of independent and previously unrelated results from Hopf algebra theory. This result states that if $A \otimes_{R} B$ is a twisted tensor product and on the vector space $B$ we have one more algebra structure denoted by $B^{\prime}$ and we have also two linear maps $\theta, \gamma: B \rightarrow A \otimes B$ satisfying a set of conditions, then we can define a new map $R^{\prime}: B^{\prime} \otimes A \rightarrow A \otimes B^{\prime}$ by a certain formula. This map turns out to be a twisting map, and we have an algebra isomorphism $A \otimes_{R^{\prime}} B^{\prime} \simeq A \otimes_{R} B$.

On the other hand, there exist important examples of "products" of "algebras" that are not twisted tensor products, a prominent example being the classical Hopf crossed product. A very general construction generalizing both the Hopf crossed product and the twisted tensor product of algebras was introduced by Brzeziński in [1]. Given an algebra $A$, a vector space $V$ endowed with a distinguished element $1_{V}$

Received by the editors December 10, 2010.

2010 Mathematics Subject Classification. Primary 16S99; Secondary 16T99.

Research partially supported by the CNCSIS project "Hopf algebras, cyclic homology and monoidal categories", contract No. 560/2009, CNCSIS code ID_69.

(C)2011 American Mathematical Society Reverts to public domain 28 years from publication 
and two linear maps $\sigma: V \otimes V \rightarrow A \otimes V$ and $R: V \otimes A \rightarrow A \otimes V$ satisfying certain conditions, Brzeziński defined an (associative unital) algebra structure on $A \otimes V$, which will be denoted in what follows by $A \otimes_{R, \sigma} V$ and called a crossed product. A twisted tensor product of algebras $A \otimes_{R} B$ is the crossed product $A \otimes_{R, \sigma} B$, where $\sigma: B \otimes B \rightarrow A \otimes B$ is given by $\sigma\left(b, b^{\prime}\right)=1_{A} \otimes b b^{\prime}$. Another example of a crossed product not discussed so far in the literature on this subject but important for us in what follows is a smash product $H \# B$ between a quasi-bialgebra $H$ and a right $H$-module algebra $B$ (this is the right-handed version of the smash product introduced in [2]; since in general $B$ is not associative, in general $H \# B$ is not a twisted tensor product of algebras).

The aim of this paper is to prove a result of the type invariance under twisting for Brzeziński's crossed products. This result arose as a common generalization of the invariance under twisting for twisted tensor products of algebras and the invariance under twisting of the quasi-Hopf smash product from [3]. Namely, if $A \otimes_{R, \sigma} V$ is a crossed product and $\theta, \gamma: V \rightarrow A \otimes V$ are linear maps, we can define certain maps $\sigma^{\prime}: V \otimes V \rightarrow A \otimes V$ and $R^{\prime}: V \otimes A \rightarrow A \otimes V$ and if some conditions are satisfied, then $A \otimes_{R^{\prime}, \sigma^{\prime}} V$ is a crossed product, isomorphic to $A \otimes_{R, \sigma} V$. After proving this result we show that it contains indeed as particular cases not only the invariance under twisting for twisted tensor products of algebras and the invariance under twisting of the quasi-Hopf smash product, but also another unrelated result, namely the equivalence of crossed products by a coalgebra proved by Brzeziński (which in turn generalizes the equivalence of Hopf crossed products).

\section{Preliminaries}

We work over a commutative field $k$. All algebras, linear spaces, etc., will be over $k$; unadorned $\otimes$ means $\otimes_{k}$. By "algebra" we always mean an associative unital algebra.

We recall from [4, 7] that, given two algebras $A, B$ and a $k$-linear map $R$ : $B \otimes A \rightarrow A \otimes B$, with notation $R(b \otimes a)=a_{R} \otimes b_{R}$, for $a \in A, b \in B$, satisfying the conditions $a_{R} \otimes 1_{R}=a \otimes 1,1_{R} \otimes b_{R}=1 \otimes b,\left(a a^{\prime}\right)_{R} \otimes b_{R}=a_{R} a_{r}^{\prime} \otimes b_{R_{r}}, a_{R} \otimes\left(b b^{\prime}\right)_{R}=$ $a_{R_{r}} \otimes b_{r} b_{R}^{\prime}$, for all $a, a^{\prime} \in A$ and $b, b^{\prime} \in B$ (where $r$ and $R$ are two different indices), if we define on $A \otimes B$ a new multiplication, by $(a \otimes b)\left(a^{\prime} \otimes b^{\prime}\right)=a a_{R}^{\prime} \otimes b_{R} b^{\prime}$, then this multiplication is associative with unit $1 \otimes 1$. In this case, the map $R$ is called a twisting map between $A$ and $B$ and the new algebra structure on $A \otimes B$ is denoted by $A \otimes_{R} B$ and called the twisted tensor product of $A$ and $B$ afforded by the map $R$.

We recall from [1] the construction of Brzeziński's crossed product:

Proposition $1.1([1])$. Let $\left(A, \mu, 1_{A}\right)$ be an (associative unital) algebra and $V$ a vector space equipped with a distinguished element $1_{V} \in V$. Then the vector space $A \otimes V$ is an associative algebra with unit $1_{A} \otimes 1_{V}$ and whose multiplication has the property that $\left(a \otimes 1_{V}\right)(b \otimes v)=a b \otimes v$, for all $a, b \in A$ and $v \in V$, if and only if there exist linear maps $\sigma: V \otimes V \rightarrow A \otimes V$ and $R: V \otimes A \rightarrow A \otimes V$ satisfying the following conditions:

$$
\begin{aligned}
& R\left(1_{V} \otimes a\right)=a \otimes 1_{V}, \quad R\left(v \otimes 1_{A}\right)=1_{A} \otimes v, \quad \forall a \in A, v \in V, \\
& \sigma\left(1_{V}, v\right)=\sigma\left(v, 1_{V}\right)=1_{A} \otimes v, \quad \forall v \in V, \\
& R \circ\left(i d_{V} \otimes \mu\right)=\left(\mu \otimes i d_{V}\right) \circ\left(i d_{A} \otimes R\right) \circ\left(R \otimes i d_{A}\right), \\
& \left(\mu \otimes i d_{V}\right) \circ\left(i d_{A} \otimes \sigma\right) \circ\left(R \otimes i d_{V}\right) \circ\left(i d_{V} \otimes \sigma\right)
\end{aligned}
$$




$$
\begin{gathered}
=\left(\mu \otimes i d_{V}\right) \circ\left(i d_{A} \otimes \sigma\right) \circ\left(\sigma \otimes i d_{V}\right), \\
\left(\mu \otimes i d_{V}\right) \circ\left(i d_{A} \otimes \sigma\right) \circ\left(R \otimes i d_{V}\right) \circ\left(i d_{V} \otimes R\right) \\
=\left(\mu \otimes i d_{V}\right) \circ\left(i d_{A} \otimes R\right) \circ\left(\sigma \otimes i d_{A}\right) .
\end{gathered}
$$

If this is the case, the multiplication of $A \otimes V$ is given explicitly by

$$
\mu_{A \otimes V}=\left(\mu_{2} \otimes i d_{V}\right) \circ\left(i d_{A} \otimes i d_{A} \otimes \sigma\right) \circ\left(i d_{A} \otimes R \otimes i d_{V}\right),
$$

where $\mu_{2}=\mu \circ\left(i d_{A} \otimes \mu\right)=\mu \circ\left(\mu \otimes i d_{A}\right)$. We denote by $A \otimes_{R, \sigma} V$ this algebra structure and call it the crossed product afforded by the data $(A, V, R, \sigma)$.

If $A \otimes_{R, \sigma} V$ is a crossed product, we introduce the Sweedler-type notation:

$$
\begin{array}{ll}
R: V \otimes A \rightarrow A \otimes V, & R(v \otimes a)=a_{R} \otimes v_{R}, \\
\sigma: V \otimes V \rightarrow A \otimes V, & \sigma\left(v, v^{\prime}\right)=\sigma_{1}\left(v, v^{\prime}\right) \otimes \sigma_{2}\left(v, v^{\prime}\right),
\end{array}
$$

for all $v, v^{\prime} \in V$ and $a \in A$. With this notation, the multiplication of $A \otimes_{R, \sigma} V$ reads

$$
(a \otimes v)\left(a^{\prime} \otimes v^{\prime}\right)=a a_{R}^{\prime} \sigma_{1}\left(v_{R}, v^{\prime}\right) \otimes \sigma_{2}\left(v_{R}, v^{\prime}\right), \quad \forall a, a^{\prime} \in A, v, v^{\prime} \in V .
$$

A twisted tensor product is a particular case of a crossed product (cf. [5]), namely, if $A \otimes_{R} B$ is a twisted tensor product of algebras, then $A \otimes_{R} B=A \otimes_{R, \sigma} B$, where $\sigma: B \otimes B \rightarrow A \otimes B$ is given by $\sigma\left(b, b^{\prime}\right)=1_{A} \otimes b b^{\prime}$, for all $b, b^{\prime} \in B$.

\section{The MAin Result And its CONSEQUences}

We can now state the invariance under the twisting theorem for crossed products:

Theorem 2.1. Let $A \otimes_{R, \sigma} V$ be a crossed product and assume we are given two linear maps $\theta, \gamma: V \rightarrow A \otimes V$, with notation $\theta(v)=v_{<-1>} \otimes v_{<0>}$ and $\gamma(v)=$ $v_{\{-1\}} \otimes v_{\{0\}}$, for all $v \in V$. Define the maps $R^{\prime}: V \otimes A \rightarrow A \otimes V$ and $\sigma^{\prime}: V \otimes V \rightarrow$ $A \otimes V$ by the formulae

$$
\begin{aligned}
R^{\prime}= & \left(\mu_{2} \otimes i d_{V}\right) \circ\left(i d_{A} \otimes i d_{A} \otimes \gamma\right) \circ\left(i d_{A} \otimes R\right) \circ\left(\theta \otimes i d_{A}\right), \\
\sigma^{\prime}= & \left(\mu \otimes i d_{V}\right) \circ\left(i d_{A} \otimes \gamma\right) \circ\left(\mu_{2} \otimes i d_{V}\right) \circ\left(i d_{A} \otimes i d_{A} \otimes \sigma\right) \\
& \circ\left(i d_{A} \otimes R \otimes i d_{V}\right) \circ(\theta \otimes \theta) .
\end{aligned}
$$

Assume that the following conditions are satisfied:

$$
\begin{aligned}
& \theta\left(1_{V}\right)=1_{A} \otimes 1_{V}, \quad \gamma\left(1_{V}\right)=1_{A} \otimes 1_{V}, \\
& v_{<-1>} v_{<0>\{-1\}} \otimes v_{<0>\{0\}}=1_{A} \otimes v, \quad \forall v \in V, \\
& v_{\{-1\}} v_{\{0\}_{<-1>}} \otimes v_{\{0\}<0>}=1_{A} \otimes v, \quad \forall v \in V, \\
& \left(\mu \otimes i d_{V}\right) \circ\left(\mu \otimes \sigma^{\prime}\right) \circ\left(i d_{A} \otimes \gamma \otimes i d_{V}\right) \circ\left(R \otimes i d_{V}\right) \circ\left(i d_{V} \otimes \gamma\right) \\
& \quad=\left(\mu \otimes i d_{V}\right) \circ\left(i d_{A} \otimes \gamma\right) \circ \sigma .
\end{aligned}
$$

Then $A \otimes_{R^{\prime}, \sigma^{\prime}} V$ is also a crossed product and we have an algebra isomorphism $A \otimes_{R^{\prime}, \sigma^{\prime}} V \simeq A \otimes_{R, \sigma} V, a \otimes v \mapsto a v_{<-1>} \otimes v_{<0>}$.

Proof. Let us note first that, in Sweedler-type notation, the maps $R^{\prime}$ and $\sigma^{\prime}$ are given by

$$
\begin{aligned}
R^{\prime}(v \otimes a)= & v_{<-1>} a_{R} v_{<0>_{R_{\{-1\}}}} \otimes v_{<0>_{R_{\{0\}}},} \\
\sigma^{\prime}(v, w)= & v_{<-1>} w_{<-1>_{R}} \sigma_{1}\left(v_{<0>_{R}}, w_{<0>}\right) \sigma_{2}\left(v_{<0>_{R}}, w_{<0>}\right)_{\{-1\}} \\
& \otimes \sigma_{2}\left(v_{<0>_{R}}, w_{<0>}\right)_{\{0\}} .
\end{aligned}
$$


We need to prove that the maps $R^{\prime}$ and $\sigma^{\prime}$ satisfy the conditions (1.1)-(1.5). The conditions (1.1) and (1.2) are very easy to prove and are left to the reader, so we concentrate on (1.3) - (1.5). We denote as usual by $R=r=\mathcal{R}=\bar{R}$ some more copies of $R$.

\section{Proof of (1.3).}

$$
\begin{aligned}
& \left(\mu \otimes i d_{V}\right) \circ\left(i d_{A} \otimes R^{\prime}\right) \circ\left(R^{\prime} \otimes i d_{A}\right)\left(v \otimes a \otimes a^{\prime}\right) \\
& =\left(\mu \otimes i d_{V}\right) \circ\left(i d_{A} \otimes R^{\prime}\right)\left(v_{<-1>} a_{R} v_{<0>_{R_{\{-1\}}}} \otimes v_{<0>_{R_{\{0\}}}} \otimes a^{\prime}\right) \\
& =v_{<-1>} a_{R} v_{<0>R_{\{-1\}}} v_{<0>_{R_{\{0\}}}} a_{r}^{\prime} v_{<0>R_{\{0\}}<0>r_{\{-1\}}} \otimes v_{<0>_{R_{\{0\}}<0>r_{\{0\}}}}
\end{aligned}
$$

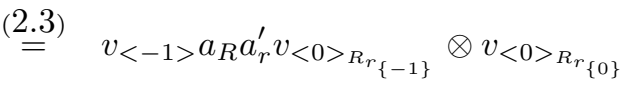

$$
\begin{aligned}
& \stackrel{1.3}{=} v_{<-1>}\left(a a^{\prime}\right)_{R} v_{<0>_{R_{\{-1\}}}} \otimes v_{<0>_{R_{\{0\}}}} \\
& =R^{\prime} \circ\left(i d_{V} \otimes \mu\right)\left(v \otimes a \otimes a^{\prime}\right), \quad \text { q.e.d. }
\end{aligned}
$$

Proof of (1.4). Note first that (1.4) and (1.5) for $R, \sigma$ may be written in Sweedlertype notation as

$$
\begin{aligned}
& \sigma_{1}(y, z)_{R} \sigma_{1}\left(x_{R}, \sigma_{2}(y, z)\right) \otimes \sigma_{2}\left(x_{R}, \sigma_{2}(y, z)\right) \\
& \quad=\sigma_{1}(x, y) \sigma_{1}\left(\sigma_{2}(x, y), z\right) \otimes \sigma_{2}\left(\sigma_{2}(x, y), z\right), \\
& a_{R_{r}} \sigma_{1}\left(v_{r}, w_{R}\right) \otimes \sigma_{2}\left(v_{r}, w_{R}\right)=\sigma_{1}(v, w) a_{R} \otimes \sigma_{2}(v, w)_{R},
\end{aligned}
$$

for all $a \in A$ and $x, y, z, v, w \in V$. Now we compute:

$$
\begin{aligned}
& \left(\mu \otimes i d_{V}\right) \circ\left(i d_{A} \otimes \sigma^{\prime}\right) \circ\left(R^{\prime} \otimes i d_{V}\right) \circ\left(i d_{V} \otimes \sigma^{\prime}\right)(x \otimes y \otimes z) \\
& =\left(\mu \otimes i d_{V}\right) \circ\left(i d_{A} \otimes \sigma^{\prime}\right) \circ\left(R^{\prime} \otimes i d_{V}\right)\left(x \otimes y_{<-1>} z_{<-1>_{R}}\right. \\
& \left.\sigma_{1}\left(y_{<0>R}, z_{<0>}\right) \sigma_{2}\left(y_{<0>_{R}}, z_{<0>}\right)_{\{-1\}} \otimes \sigma_{2}\left(y_{<0>_{R}}, z_{<0>}\right)_{\{0\}}\right) \\
& =\left(\mu \otimes i d_{V}\right) \circ\left(i d_{A} \otimes \sigma^{\prime}\right)\left(x _ { < - 1 > } \left[y_{<-1>} z_{<-1>_{R}} \sigma_{1}\left(y_{<0>R}, z_{<0>}\right)\right.\right. \\
& \left.\left.\sigma_{2}\left(y_{<0>_{R}}, z_{<0>}\right)_{\{-1\}}\right]_{r} x_{<0>_{r_{\{-1\}}}} \otimes x_{<0>_{r_{\{0\}}}} \otimes \sigma_{2}\left(y_{<0>_{R}}, z_{<0>}\right)_{\{0\}}\right) \\
& =x_{<-1>}\left[y_{<-1>} z_{<-1>_{R}} \sigma_{1}\left(y_{<0>R}, z_{<0>}\right) \sigma_{2}\left(y_{<0>_{R}}, z_{<0>}\right)_{\{-1\}}\right]_{r} \\
& x_{<0>r_{\{-1\}}} x_{<0>r_{\{0\}<-1>}} \sigma_{2}\left(y_{<0>R}, z_{<0>}\right)_{\{0\}_{<-1>\mathcal{R}}}
\end{aligned}
$$

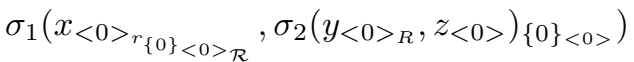

$$
\begin{aligned}
& \sigma_{2}\left(x_{<0>_{r_{\{0\}}<0>\mathcal{R}}}, \sigma_{2}\left(y_{<0>R}, z_{<0>}\right)_{\{0\}<0>}\right)_{\{-1\}} \\
& \otimes \sigma_{2}\left(x_{<0>_{r_{\{0\}}<0>\mathcal{R}}}, \sigma_{2}\left(y_{<0>_{R}}, z_{<0>}\right)_{\{0\}<0>}\right)_{\{0\}} \\
& \stackrel{2.3}{=} x_{<-1>}\left[y_{<-1>} z_{<-1>_{R}} \sigma_{1}\left(y_{<0>_{R}}, z_{<0>}\right) \sigma_{2}\left(y_{<0>_{R}}, z_{<0>}\right)_{\{-1\}}\right]_{r}
\end{aligned}
$$

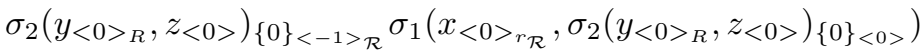

$$
\begin{aligned}
& \sigma_{2}\left(x_{<0>_{r_{\mathcal{R}}}}, \sigma_{2}\left(y_{<0>R}, z_{<0>}\right)_{\{0\}<0>}\right)_{\{-1\}}
\end{aligned}
$$

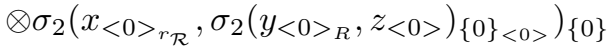


1.3

$$
\begin{aligned}
& x_{<-1>}\left[y_{<-1>} z_{<-1>_{R}} \sigma_{1}\left(y_{<0>_{R}}, z_{<0>}\right)\right]_{\bar{R}} \sigma_{2}\left(y_{<0>_{R}}, z_{<0>}\right)_{\{-1\}_{r}}
\end{aligned}
$$

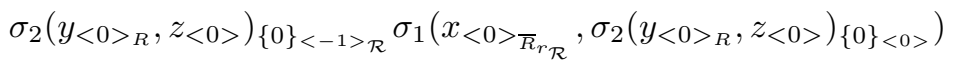

$$
\begin{aligned}
& \sigma_{2}\left(x_{<0>\bar{R}_{r_{\mathcal{R}}}}, \sigma_{2}\left(y_{<0>_{R}}, z_{<0>}\right)_{\{0\}<0>}\right)_{\{-1\}}
\end{aligned}
$$

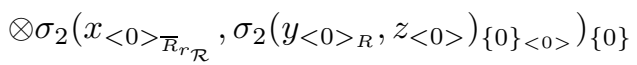

1.3

$$
\begin{aligned}
& x_{<-1>}\left[y_{<-1>} z_{<-1>R} \sigma_{1}\left(y_{<0>_{R}}, z_{<0>}\right)\right]_{\bar{R}} \\
& {\left[\sigma_{2}\left(y_{<0>_{R}}, z_{<0>}\right)_{\{-1\}} \sigma_{2}\left(y_{<0>_{R}}, z_{<0>}\right)_{\{0\}<-1>}\right]_{r}} \\
& \sigma_{1}\left(x_{<0>_{\bar{R}_{r}}}, \sigma_{2}\left(y_{<0>_{R}}, z_{<0>}\right)_{\{0\}_{<0>}}\right) \\
& \sigma_{2}\left(x_{<0 \overline{\bar{R}}_{r}}, \sigma_{2}\left(y_{<0>_{R}}, z_{<0>}\right)_{\{0\}_{<0>}}\right)_{\{-1\}} \\
& \otimes \sigma_{2}\left(x_{<0>_{\bar{R}_{r}}}, \sigma_{2}\left(y_{<0>_{R}}, z_{<0>}\right)_{\{0\}_{<0>}}\right)_{\{0\}}
\end{aligned}
$$

$2.3,1.1$

$$
\begin{aligned}
& x_{<-1>}\left[y_{<-1>} z_{<-1>_{R}} \sigma_{1}\left(y_{<0>_{R}}, z_{<0>}\right)\right]_{\bar{R}} \sigma_{1}\left(x_{<0>_{\bar{R}}}, \sigma_{2}\left(y_{<0>_{R}}, z_{<0>}\right)\right) \\
& \sigma_{2}\left(x_{<0>_{\bar{R}}}, \sigma_{2}\left(y_{<0>_{R}}, z_{<0>}\right)\right)_{\{-1\}} \otimes \sigma_{2}\left(x_{<0>_{\bar{R}}}, \sigma_{2}\left(y_{<0>_{R}}, z_{<0>}\right)\right)_{\{0\}}
\end{aligned}
$$

$$
\begin{aligned}
& x_{<-1>} y_{<-1>_{\bar{R}}} z_{<-1>_{R_{r}}} \sigma_{1}\left(y_{<0>_{R}}, z_{<0>}\right)_{\mathcal{R}} \\
& \sigma_{1}\left(x_{<0>_{\bar{R}_{r_{\mathcal{R}}}}}, \sigma_{2}\left(y_{<0>_{R}}, z_{<0>}\right)\right) \\
& \sigma_{2}\left(x_{<0>_{\bar{R}_{r_{\mathcal{R}}}}}, \sigma_{2}\left(y_{<0>_{R}}, z_{<0>}\right)\right)_{\{-1\}} \\
& \otimes \sigma_{2}\left(x_{<0>>_{\bar{R}_{r_{\mathcal{R}}}}}, \sigma_{2}\left(y_{<0>R}, z_{<0>}\right)\right)_{\{0\}}
\end{aligned}
$$

$$
\begin{aligned}
& x_{<-1>} y_{<-1>\bar{R}_{\bar{R}}} z_{<-1>_{R_{r}}} \sigma_{1}\left(x_{<0 \overline{\bar{R}}_{r}}, y_{<0>_{R}}\right) \\
& \sigma_{1}\left(\sigma_{2}\left(x_{<0>_{\bar{R}_{r}}}, y_{<0>_{R}}\right), z_{<0>}\right) \\
& \sigma_{2}\left(\sigma_{2}\left(x_{<0 \overline{\bar{R}}_{r}}, y_{<0>_{R}}\right), z_{<0>}\right)_{\{-1\}} \\
& \otimes \sigma_{2}\left(\sigma_{2}\left(x_{<0>_{\bar{R}_{r}}}, y_{<0>_{R}}\right), z_{<0>}\right)_{\{0\}},
\end{aligned}
$$

$\left(\mu \otimes i d_{V}\right) \circ\left(i d_{A} \otimes \sigma^{\prime}\right) \circ\left(\sigma^{\prime} \otimes i d_{V}\right)(x \otimes y \otimes z)$

$$
\begin{aligned}
& =\left(\mu \otimes i d_{V}\right) \circ\left(i d_{A} \otimes \sigma^{\prime}\right)\left(x_{<-1>} y_{<-1>_{\bar{R}}} \sigma_{1}\left(x_{<0>_{\bar{R}}}, y_{<0>}\right)\right. \\
& \left.\sigma_{2}\left(x_{<0>\bar{R}}, y_{<0>}\right)_{\{-1\}} \otimes \sigma_{2}\left(x_{<0>\bar{R}}, y_{<0>}\right)_{\{0\}} \otimes z\right) \\
& =x_{<-1>} y_{<-1>\bar{R}} \sigma_{1}\left(x_{<0>_{\bar{R}}}, y_{<0>}\right) \sigma_{2}\left(x_{<0>_{\bar{R}}}, y_{<0>}\right)_{\{-1\}} \\
& \sigma_{2}\left(x_{<0>_{\bar{R}}}, y_{<0>}\right)_{\{0\}<-1>} z_{<-1>_{R}} \sigma_{1}\left(\sigma_{2}\left(x_{<0>_{\bar{R}}}, y_{<0>}\right)_{\left.\{0\}_{<0>_{R}}, z_{<0>}\right)}\right. \\
& \sigma_{2}\left(\sigma_{2}\left(x_{<0>_{\bar{R}}}, y_{<0>}\right)_{\{0\}_{<0>R}}, z_{<0>}\right)_{\{-1\}} \\
& \otimes \sigma_{2}\left(\sigma_{2}\left(x_{<0>\bar{R}}, y_{<0>}\right)_{\{0\}_{<0>}}, z_{<0>}\right)_{\{0\}} \\
& \stackrel{2.3}{=} x_{<-1>} y_{<-1>_{\bar{R}}} \sigma_{1}\left(x_{<0>_{\bar{R}}}, y_{<0>}\right) z_{<-1>_{R}} \sigma_{1}\left(\sigma_{2}\left(x_{<0>_{\bar{R}}}, y_{<0>}\right)_{R}, z_{<0>}\right) \\
& \sigma_{2}\left(\sigma_{2}\left(x_{<0>\bar{R}}, y_{<0>}\right)_{R}, z_{<0>}\right)_{\{-1\}} \otimes \sigma_{2}\left(\sigma_{2}\left(x_{<0>\bar{R}}, y_{<0>}\right)_{R}, z_{<0>}\right)_{\{0\}} \\
& \stackrel{1.5}{=} \\
& x_{<-1>} y_{<-1 \bar{R}_{\bar{R}}} z_{<-1>_{R_{r}}} \sigma_{1}\left(x_{<0>_{\bar{R}_{r}}}, y_{<0>_{R}}\right) \\
& \sigma_{1}\left(\sigma_{2}\left(x_{<0>_{\bar{R}_{r}}}, y_{<0>_{R}}\right), z_{<0>}\right) \\
& \sigma_{2}\left(\sigma_{2}\left(x_{<0>_{\bar{R}_{r}}}, y_{<0>_{R}}\right), z_{<0>}\right)_{\{-1\}} \\
& \otimes \sigma_{2}\left(\sigma_{2}\left(x_{<0>_{\bar{R}_{r}}}, y_{<0>_{R}}\right), z_{<0>}\right)_{\{0\}},
\end{aligned}
$$

and we see that the two terms coincide. 
Proof of (1.5). Let $v, w \in V$ and $a \in A$. We compute:

$$
\begin{aligned}
& \left(\mu \otimes i d_{V}\right) \circ\left(i d_{A} \otimes \sigma^{\prime}\right) \circ\left(R^{\prime} \otimes i d_{V}\right) \circ\left(i d_{V} \otimes R^{\prime}\right)(v \otimes w \otimes a) \\
& =\left(\mu \otimes i d_{V}\right) \circ\left(i d_{A} \otimes \sigma^{\prime}\right) \circ\left(R^{\prime} \otimes i d_{V}\right)\left(v \otimes w_{<-1>} a_{r} w_{<0>_{r_{\{-1\}}}} \otimes w_{<0>_{r_{\{0\}}}}\right) \\
& =\left(\mu \otimes i d_{V}\right) \circ\left(i d_{A} \otimes \sigma^{\prime}\right)\left(v_{<-1>}\left(w_{<-1>} a_{r} w_{<0>_{r_{\{-1\}}}}\right)_{R} v_{<0>_{R_{\{-1\}}}}\right. \\
& \otimes v_{<0>_{R_{\{0\}}}} \otimes w_{\left.<0>_{r_{\{0\}}}\right)} \\
& =v_{<-1>}\left(w_{<-1>} a_{r} w_{<0>_{r_{\{-1\}}}}\right)_{R} v_{<0>_{R_{\{-1\}}}} \sigma_{1}^{\prime}\left(v_{<0>_{R_{\{0\}}}}, w_{<0>_{r_{\{0\}}}}\right) \\
& \otimes \sigma_{2}^{\prime}\left(v_{<0>R_{\{0\}}}, w_{<0>r_{\{0\}}}\right) \\
& 1.3
\end{aligned}
$$

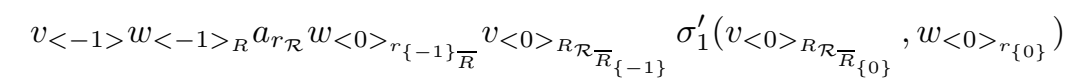

$$
\begin{aligned}
& \otimes \sigma_{2}^{\prime}\left(v_{<0>_{R_{\mathcal{R}}}}, w_{<0>_{r_{\{0\}}}}\right) \\
& v_{<-1>} w_{<-1>_{R}} a_{r_{\mathcal{R}}} \sigma_{1}\left(v_{<0>_{R_{\mathcal{R}}}}, w_{<0>_{r}}\right) \sigma_{2}\left(v_{<0>_{R_{\mathcal{R}}}}, w_{<0>_{r}}\right)_{\{-1\}} \\
& \otimes \sigma_{2}\left(v_{<0>_{R_{\mathcal{R}}}}, w_{<0>_{r}}\right)_{\{0\}},
\end{aligned}
$$

$\left(\mu \otimes i d_{V}\right) \circ\left(i d_{A} \otimes R^{\prime}\right) \circ\left(\sigma^{\prime} \otimes i d_{A}\right)(v \otimes w \otimes a)$

$$
\begin{aligned}
& =\left(\mu \otimes i d_{V}\right) \circ\left(i d_{A} \otimes R^{\prime}\right)\left(v_{<-1>} w_{<-1>_{R}} \sigma_{1}\left(v_{<0>_{R}}, w_{<0>}\right)\right. \\
& \left.\sigma_{2}\left(v_{<0>_{R}}, w_{<0>}\right)_{\{-1\}} \otimes \sigma_{2}\left(v_{<0>_{R}}, w_{<0>}\right)_{\{0\}} \otimes a\right) \\
& =v_{<-1>} w_{<-1>_{R}} \sigma_{1}\left(v_{<0>_{R}}, w_{<0>}\right) \sigma_{2}\left(v_{<0>_{R}}, w_{<0>}\right)_{\{-1\}} \\
& \sigma_{2}\left(v_{<0>R}, w_{<0>}\right)_{\{0\}_{<-1>}} a_{r} \sigma_{2}\left(v_{<0>R}, w_{<0>}\right)_{\{0\}_{<0>r_{\{-1\}}}} \\
& \otimes \sigma_{2}\left(v_{<0>_{R}}, w_{<0>}\right)\{0\}_{<0>r_{\{0\}}} \\
& 2.3 \\
& v_{<-1>} w_{<-1>_{R}} \sigma_{1}\left(v_{<0>_{R}}, w_{<0>}\right) a_{r} \sigma_{2}\left(v_{<0>_{R}}, w_{<0>}\right)_{r_{\{-1\}}} \\
& \otimes \sigma_{2}\left(v_{<0>_{R}}, w_{<0>}\right)_{r_{\{0\}}} \\
& \stackrel{1.5}{=} v_{<-1>} w_{<-1>_{R}} a_{r_{\mathcal{R}}} \sigma_{1}\left(v_{<0>_{R_{\mathcal{R}}}}, w_{<0>_{r}}\right) \sigma_{2}\left(v_{<0>_{R_{\mathcal{R}}}}, w_{<0>_{r}}\right)_{\{-1\}} \\
& \otimes \sigma_{2}\left(v_{<0>_{R_{\mathcal{R}}}}, w_{<0>_{r}}\right)_{\{0\}},
\end{aligned}
$$

and we see that the two terms coincide. Thus, $A \otimes_{R^{\prime}, \sigma^{\prime}} V$ is indeed a crossed product.

We now prove that the map $\varphi: A \otimes_{R^{\prime}, \sigma^{\prime}} V \rightarrow A \otimes_{R, \sigma} V, \varphi(a \otimes v)=a v_{<-1>} \otimes v_{<0>}$, is an algebra isomorphism. First, using (2.2) and (2.3), it is easy to see that $\varphi$ is bijective, with inverse given by $a \otimes v \mapsto a v_{\{-1\}} \otimes v_{\{0\}}$. It is obvious that $\varphi(1 \otimes 1)=1 \otimes 1$, so we only have to prove that $\varphi$ is multiplicative. We compute:

$$
\begin{aligned}
& \varphi\left((a \otimes v)\left(a^{\prime} \otimes v^{\prime}\right)\right) \\
& =\varphi\left(a a_{R^{\prime}}^{\prime} \sigma_{1}^{\prime}\left(v_{R^{\prime}}, v^{\prime}\right) \otimes \sigma_{2}^{\prime}\left(v_{R^{\prime}}, v^{\prime}\right)\right) \\
& =\varphi\left(a v_{<-1>} a_{R}^{\prime} v_{<0>_{R_{\{-1\}}}} \sigma_{1}^{\prime}\left(v_{<0>_{R_{\{0\}}}}, v^{\prime}\right) \otimes \sigma_{2}^{\prime}\left(v_{<0>_{R_{\{0\}}}}, v^{\prime}\right)\right) \\
& =\varphi\left(a v_{<-1>} a_{R}^{\prime} v_{<0>_{R_{\{-1\}}}} v_{<0>_{R_{\{0\}}}} v_{<-1>}^{\prime} \sigma_{r} \sigma_{1}\left(v_{<0>_{R_{\{0\}}}, 0>r}, v_{<0>}^{\prime}\right)\right. \\
& \left.\sigma_{2}\left(v_{<0>_{R_{\{0\}}<0>r}}, v_{<0>}^{\prime}\right)_{\{-1\}} \otimes \sigma_{2}\left(v_{<0>_{R_{\{0\}}}, 0>r}, v_{<0>}^{\prime}\right)_{\{0\}}\right) \\
& \stackrel{2.3}{=} \varphi\left(a v_{<-1>} a_{R}^{\prime} v_{<-1>r}^{\prime} \sigma_{1}\left(v_{<0>_{R}}, v_{<0>}^{\prime}\right)\right. \\
& \left.\sigma_{2}\left(v_{<0>_{R_{r}}}, v_{<0>}^{\prime}\right)_{\{-1\}} \otimes \sigma_{2}\left(v_{<0>_{R_{r}}}, v_{<0>}^{\prime}\right)_{\{0\}}\right)
\end{aligned}
$$




$$
\begin{array}{ll}
= & a v_{<-1>} a_{R}^{\prime} v_{<-1>r}^{\prime} \sigma_{1}\left(v_{<0>_{R}}, v_{<0>}^{\prime}\right) \sigma_{2}\left(v_{<0>_{R}}, v_{<0>}^{\prime}\right)_{\{-1\}} \\
& \sigma_{2}\left(v_{<0 R_{r}}, v_{<0>}^{\prime}\right)\{0\}_{<-1>} \otimes \sigma_{2}\left(v_{<0>_{r}}, v_{<0>}^{\prime}\right)\{0\}_{<0>} \\
\underline{\underline{2.3}} & a v_{<-1>} a_{R}^{\prime} v_{<-1>r}^{\prime} \sigma_{1}\left(v_{<0>_{R}}, v_{<0>}^{\prime}\right) \otimes \sigma_{2}\left(v_{<0>_{R}}, v_{<0>}^{\prime}\right) \\
\underline{\underline{1.3}} & a v_{<-1>}\left(a^{\prime} v_{<-1>}^{\prime}\right)_{R} \sigma_{1}\left(v_{<0>R}, v_{<0>}^{\prime}\right) \otimes \sigma_{2}\left(v_{<0>_{R}}, v_{<0>}^{\prime}\right) \\
= & \left(a v_{<-1>} \otimes v_{<0>}\right)\left(a^{\prime} v_{<-1>}^{\prime} \otimes v_{<0>}^{\prime}\right) \\
= & \varphi(a \otimes v) \varphi\left(a^{\prime} \otimes v^{\prime}\right),
\end{array}
$$

finishing the proof.

We now explain that Theorem 2.1 indeed generalizes the invariance under twisting for twisted tensor products of algebras proved in Theorem 4.8 in [6], which we first recall:

Theorem 2.2 ([6]). Let $A \otimes_{R} B$ be a twisted tensor product of algebras and denote the multiplication of $B$ by $b \otimes b^{\prime} \mapsto b b^{\prime}$. Assume that on the vector space $B$ we have one more algebra structure, denoted by $B^{\prime}$, with the same unit as $B$ and multiplication denoted by $b \otimes b^{\prime} \mapsto b * b^{\prime}$. Assume that we are given two linear maps $\theta, \gamma: B \rightarrow A \otimes B$, with notation $\theta(b)=b_{<-1>} \otimes b_{<0>}$ and $\gamma(b)=b_{\{-1\}} \otimes b_{\{0\}}$, such that $\theta$ is an algebra map from $B^{\prime}$ to $A \otimes_{R} B, \gamma(1)=1 \otimes 1$ and the following relations are satisfied, for all $b, b^{\prime} \in B$ :

$$
\begin{aligned}
& \gamma\left(b b^{\prime}\right)=b_{\{-1\}_{R}}^{\prime} b_{R_{\{-1\}}} \otimes b_{R_{\{0\}}} * b_{\{0\}}^{\prime}, \\
& b_{<-1>} b_{<0>_{\{-1\}}} \otimes b_{<0>\{0\}}=1 \otimes b, \\
& b_{\{-1\}} b_{\{0\}<-1>} \otimes b_{\{0\}<0>}=1 \otimes b .
\end{aligned}
$$

Then the map $R^{\prime}: B^{\prime} \otimes A \rightarrow A \otimes B^{\prime}, R^{\prime}(b \otimes a)=b_{<-1>} a_{R} b_{<0>_{R_{\{-1\}}}} \otimes b_{<0>_{R_{\{0\}}}}$, is a twisting map and we have an algebra isomorphism $A \otimes_{R^{\prime}} B^{\prime} \simeq A \otimes_{R} B, a \otimes b \mapsto$ $a b_{<-1>} \otimes b_{<0>}$.

We want to see how Theorem 2.1 generalizes Theorem 2.2. We begin with the data $A, B, B^{\prime}, R, \theta, \gamma$ as in the hypothesis of Theorem 2.2 , consider the map $\sigma: B \otimes B \rightarrow A \otimes B, \sigma\left(b, b^{\prime}\right)=1_{A} \otimes b b^{\prime}$, for all $b, b^{\prime} \in B$, so we have the crossed product $A \otimes_{R, \sigma} B$ with $A \otimes_{R} B=A \otimes_{R, \sigma} B$ and the maps $\theta, \gamma$ and so we can define the maps $R^{\prime}$ and $\sigma^{\prime}$ as in Theorem 2.1 (of course, $V$ in Theorem 2.1] is $B$ as vector spaces). Obviously, the formula for $R^{\prime}$ in Theorem 2.1 is the same as the one for $R^{\prime}$ in Theorem 2.2. We want to see what $\sigma^{\prime}$ looks like. By using the fact that $\theta$ is an algebra map from $B^{\prime}$ to $A \otimes_{R} B$ and the formula $\sigma\left(b, b^{\prime}\right)=1_{A} \otimes b b^{\prime}$, an easy computation shows that we have $\sigma^{\prime}\left(b, b^{\prime}\right)=1_{A} \otimes b * b^{\prime}$, for all $b, b^{\prime} \in B$. Then one can easily see that the relations (2.1) -(2.4) hold (note that (2.4) reduces to (2.7)). Thus, all hypotheses of Theorem 2.1 are fulfilled, so we have the crossed product $A \otimes_{R^{\prime}, \sigma^{\prime}} B$ and the algebra isomorphism $A \otimes_{R^{\prime}, \sigma^{\prime}} B \simeq A \otimes_{R, \sigma} B$. But since $\sigma^{\prime}$ is defined by $\sigma^{\prime}\left(b, b^{\prime}\right)=1_{A} \otimes b * b^{\prime}$, it is obvious that the multiplication of $A \otimes_{R^{\prime}, \sigma^{\prime}} B$ is exactly the one of $A \otimes_{R^{\prime}} B^{\prime}$, i.e. $A \otimes_{R^{\prime}, \sigma^{\prime}} B \equiv A \otimes_{R^{\prime}} B^{\prime}$, and so we obtain the algebra isomorphism $A \otimes_{R^{\prime}} B^{\prime} \simeq A \otimes_{R} B$ as in Theorem 2.2.

We explain now that Theorem 2.1 generalizes the invariance under twisting for (right) quasi-Hopf smash products ([3]), which we recall first (we use terminology and notation as in [3]).

Let $H$ be a quasi-bialgebra with associator $\Phi$ and $F \in H \otimes H$ a gauge transformation, with notation $F=F^{1} \otimes F^{2}$ and $F^{-1}=G^{1} \otimes G^{2}$. Consider the Drinfeld 
twist of $H$, denoted by $H_{F}$, which is a quasi-bialgebra having the same underlying vector space, multiplication, unit and counit as $H$ and comultiplication and associator defined by

$$
\Delta_{F}(h)=F \Delta(h) F^{-1}, \quad \Phi_{F}=(1 \otimes F)(i d \otimes \Delta)(F) \Phi(\Delta \otimes i d)\left(F^{-1}\right)\left(F^{-1} \otimes 1\right) .
$$

We denote what follows by $\Delta_{F}(h)=h_{(1)} \otimes h_{(2)}, \Phi_{F}=\tilde{X}^{1} \otimes \tilde{X}^{2} \otimes \tilde{X}^{3}, \Phi_{F}^{-1}=$ $\tilde{x}^{1} \otimes \tilde{x}^{2} \otimes \tilde{x}^{3}$.

Let $B$ be a right $H$-module algebra (an algebra in the monoidal category of right $H$-modules), so we can consider the (right) smash product $H \# B$, which is an associative algebra having $H \otimes B$ as underlying vector space, multiplication $(h \# b)\left(h^{\prime} \# b^{\prime}\right)=h h_{1}^{\prime} x^{1} \#\left(b \cdot h_{2}^{\prime} x^{2}\right)\left(b^{\prime} \cdot x^{3}\right)$ and unit $1_{H} \# 1_{B}$ (we denoted as usual $\Phi=X^{1} \otimes X^{2} \otimes X^{3}$ and $\Phi^{-1}=x^{1} \otimes x^{2} \otimes x^{3}$ the associator of $H$ and its inverse).

Proposition 2.3 ([3]). If we introduce on $B$ another multiplication by $b * b^{\prime}=$ $\left(b \cdot F^{1}\right)\left(b^{\prime} \cdot F^{2}\right)$ and denote this structure by ${ }_{F} B$, then ${ }_{F} B$ becomes a right $H_{F^{-}}$ module algebra with the same unit and $H$-action as for $B$ and we have an algebra isomorphism $H_{F} \#_{F} B \simeq H \# B, h \# b \mapsto h F^{1} \# b \cdot F^{2}$.

We want to see that Proposition 2.3 is a particular case of Theorem 2.1. Since $H \# B$ is an associative algebra with unit $1_{H} \# 1_{B}$ and its multiplication satisfies $\left(h \# 1_{B}\right)\left(h^{\prime} \# b^{\prime}\right)=h h^{\prime} \# b^{\prime}$, for all $h, h^{\prime} \in H$ and $b \in B$, it follows that $H \# B$ is a crossed product, namely $H \# B=H \otimes_{R, \sigma} B$, where the maps $R, \sigma$ are defined, for all $b, b^{\prime} \in B$ and $h \in H$, by $R: B \otimes H \rightarrow H \otimes B, R(b \otimes h)=h_{1} \otimes b \cdot h_{2}$ and $\sigma: B \otimes B \rightarrow H \otimes B, \sigma\left(b, b^{\prime}\right)=x^{1} \otimes\left(b \cdot x^{2}\right)\left(b^{\prime} \cdot x^{3}\right)$. Similarly, we have $H_{F} \#_{F} B=$ $H \otimes_{R_{F}, \sigma_{F}} B$, where $R_{F}(b \otimes h)=h_{(1)} \otimes b \cdot h_{(2)}$ and $\sigma_{F}\left(b, b^{\prime}\right)=\tilde{x}^{1} \otimes\left(b \cdot \tilde{x}^{2}\right) *\left(b^{\prime} \cdot \tilde{x}^{3}\right)$.

If we define $\theta, \gamma: B \rightarrow H \otimes B$ by $\theta(b)=F^{1} \otimes b \cdot F^{2}$ and $\gamma(b)=G^{1} \otimes b \cdot G^{2}$, for all $b \in B$, then one can check that the hypotheses of Theorem 2.1 are fulfilled for the crossed product $H \otimes_{R, \sigma} B$ and the maps $\theta, \gamma$. It is also easy to see that the maps $R^{\prime}, \sigma^{\prime}$ given by Theorem 2.1 coincide exactly to the maps $R_{F}$ and respectively $\sigma_{F}$, thus Theorem 2.1 implies $H \otimes_{R_{F}, \sigma_{F}} B \simeq H \otimes_{R, \sigma} B$, that is, $H_{F} \#_{F} B \simeq H \# B$ (and obviously the explicit isomorphisms given by Theorem 2.1 and Proposition 2.3 coincide).

We now briefly explain how Theorem 2.1 generalizes the equivalence of crossed products by a coalgebra proved in [1, Proposition 3.1. We recall first the framework in 11. Let $P$ be an algebra, $C$ a coalgebra with a fixed group-like element $e$, let $\left(P, C, \Psi, e, \Psi^{C}\right)$ be an entwining data (see 11 for the definition) and consider the algebra $A=P_{e}^{c o(C)}$. Assume that there exist linear maps $f: C \otimes C \rightarrow A$, $F: C \otimes P \rightarrow P$, satisfying a certain set of conditions. If we define

$$
\begin{aligned}
& R_{F}: C \otimes A \rightarrow A \otimes C, \quad R_{F}=\left(F \otimes i d_{C}\right) \circ\left(i d_{C} \otimes \Psi\right) \circ\left(\Delta \otimes i d_{A}\right), \\
& \sigma_{f}: C \otimes C \rightarrow A \otimes C, \quad \sigma_{f}=\left(f \otimes i d_{C}\right) \circ\left(i d_{C} \otimes \Psi^{C}\right) \circ\left(\Delta \otimes i d_{C}\right),
\end{aligned}
$$

then $A \otimes_{R_{F}, \sigma_{f}} C$ is a crossed product, denoted by $A \rtimes_{F, f} C$ and called a crossed product by a coalgebra (see [1, Proposition 2.2 for details).

Moreover assume that $\nu: C \rightarrow A$ is a convolution invertible map (with convolution inverse denoted by $\nu^{-1}$ ) such that $\nu(e)=1$ and

$$
\begin{aligned}
\Psi_{23}^{C} \circ \Psi_{12} \circ\left(i d_{C} \otimes \nu \otimes i d_{C}\right) \circ\left(i d_{C} \otimes \Delta\right) \\
=\left(\nu \otimes i d_{C} \otimes i d_{C}\right) \circ\left(\Delta \otimes i d_{C}\right) \otimes \Psi^{C} .
\end{aligned}
$$

Define maps $F_{\nu}: C \otimes P \rightarrow P, f_{\nu}: C \otimes C \rightarrow A$ by some explicit formulae given in [1], Proposition 3.1. Then $A \rtimes_{F_{\nu}, f_{\nu}} C$ is a crossed product by a coalgebra, isomorphic 
to $A \rtimes_{F, f} C$ as algebras (this is the equivalence of crossed products by a coalgebra proved in [1, Proposition 3.1).

We want to see that this result is a particular case of Theorem 2.1. In the hypotheses of [1, Proposition 3.1, consider the linear maps $\theta, \gamma: C \rightarrow A \otimes C$, $\theta(c)=\nu\left(c_{1}\right) \otimes c_{2}, \gamma(c)=\nu^{-1}\left(c_{1}\right) \otimes c_{2}$. One can check that for the maps $\theta, \gamma$ and the crossed product $A \otimes_{R_{F}, \sigma_{f}} C$, the hypotheses of Theorem 2.1 are fulfilled (for (2.4) one has to use the relation (2.10) ). Thus, we can apply Theorem 2.1 which gives the maps $R_{F}^{\prime}$ and $\sigma_{f}^{\prime}$ and the algebra isomorphism $A \otimes_{R_{F}^{\prime}, \sigma_{f}^{\prime}} C \simeq A \otimes_{R_{F}, \sigma_{f}} C$. On the other hand, a straightforward computation shows that $R_{F_{\nu}}=R_{F}^{\prime}$ and

$\sigma_{f_{\nu}}=\sigma_{f}^{\prime}$. Thus, from Theorem 2.1 we obtain $A \rtimes_{F_{\nu}, f_{\nu}} C=A \otimes_{R_{F_{\nu}}, \sigma_{f_{\nu}}} C=$ $A \otimes_{R_{F}^{\prime}, \sigma_{f}^{\prime}} C \simeq A \otimes_{R_{F}, \sigma_{f}} C=A \rtimes_{F, f} C$, q.e.d.

\section{REFERENCES}

[1] T. Brzeziński, Crossed products by a coalgebra, Comm. Algebra 25 (1997), 3551-3575. MR.1468823 (98i:16034)

[2] D. Bulacu, F. Panaite and F. Van Oystaeyen, Quasi-Hopf algebra actions and smash products, Comm. Algebra 28 (2000), 631-651. MR.1736752 (2000m:16048)

[3] D. Bulacu, F. Panaite and F. Van Oystaeyen, Generalized diagonal crossed products and smash products for quasi-Hopf algebras. Applications, Comm. Math. Phys. 266 (2006), 355399. MR2238882 (2007e:16032)

[4] A. Cap, H. Schichl and J. Vanzura, On twisted tensor products of algebras, Comm. Algebra 23 (1995), 4701-4735. MR.1352565 (96k:16039)

[5] C. Di Luigi, J. A. Guccione and J. J. Guccione, Brzeziński's crossed products and braided Hopf crossed products, Comm. Algebra 32 (2004), 3563-3580. MR 2097479 (2005i:16077)

[6] P. Jara Martínez, J. López Peña, F. Panaite and F. Van Oystaeyen, On iterated twisted tensor products of algebras, Internat. J. Math. 19 (2008), 1053-1101. MR2458561 (2009h:16041)

[7] A. Van Daele and S. Van Keer, The Yang-Baxter and pentagon equation, Compositio Math. 91 (1994), 201-221. MR.1273649(95c:16052)

Institute of Mathematics, Romanian Academy, P.O. Box 1-764, RO-014700, Bucharest, ROMANIA

E-mail address: Florin.Panaite@imar.ro 